\title{
Shear bond strength of different retainer wires and bonding adhesives in consideration of the pretreatment process
}

\author{
Claudia Reicheneder ${ }^{1}$, Bernd Hofrichter ${ }^{1}$, Andreas Faltermeier ${ }^{1}$, Peter Proff ${ }^{1}$, Carsten Lippold ${ }^{2^{*}}$ and Christian Kirschneck $^{1}$
}

\begin{abstract}
Introduction: We aimed to compare the shear bond strength (SBS) of three different retainer wires and three different bonding adhesives in consideration of the pretreatment process of enamel surface sandblasting.

Methods: 400 extracted bovine incisors were divided into 10 groups of 20 paired specimens each. 10 specimens of each group were pretreated by enamel sandblasting. The retainer wires Bond-A-Braid ${ }^{\mathrm{T}}$, GAC-Wildcat ${ }^{\oplus}$-Twistflex and everStick ${ }^{\oplus} \mathrm{ORTHO}$ were bonded to the teeth with the adhesives Transbond ${ }^{\mathrm{TM}}{ }^{-L R}$, Tetric-EvoFlow ${ }^{\mathrm{TM}}$ and Stick ${ }^{\oplus}$ FOW and then debonded measuring the SBS.

Results: While sandblasting generally increased SBS for all tested combinations, the retainer wires bonded with Transbond ${ }^{T M}-$ LR showed the highest SBS both with and without prior sandblasting. Significantly lower SBS were found for Tetric-EvoFlow ${ }^{\mathrm{TM}}$ that were comparable to those for everStick ${ }^{\circledR} \mathrm{ORTHO}$.

Conclusions: Pretreatment of enamel surfaces by sandblasting increased the SBS of all retainer-wires. Transbond ${ }^{\mathrm{TM}}$-LR showed the best results compared to Tetric-EvoFlow ${ }^{\mathrm{TM}}$ and everStick ${ }^{\circledR} \mathrm{ORTHO}$, while all combinations used provided sufficient bonding strengths for clinical use.
\end{abstract}

Keywords: Retainer, Wire, Adhesive, Sandblasting, Shear bond strength

\section{Introduction}

Tooth movement due to the persisting imbalance of operating forces is a major problem after orthodontic treatment $[1,2]$. Stabilisation of orthodontic treatment outcomes is necessary to ensure the success of such treatments and to prevent teeth from moving back into their former position. After every orthodontic treatment, retention is essential to maintain therapy outcomes and to avoid relapse. One option is the use of retainer systems, whose positive effects have been shown before [1,2].

Two different retainer systems, fixed and removable retainers, are used in clinical practise. Removable retainers have the disadvantages of aging, reduced wearing comfort such as impaired patient speech, and their clinical success depends on sufficient patient compliance [3]. Mainly fixed retainers guarantee the stability of anterior teeth, since

\footnotetext{
*Correspondence: lippold@uni-muenster.de

${ }^{2}$ Department of Orthodontics, University of Muenster, Waldeyerstraße 30, 48149 Muenster, Germany

Full list of author information is available at the end of the article
}

they require little patient compliance. Bonded lingual retainers have become increasing popular as method of retention since the late 1970 s, particularly in the mandibular incisor area [4]. Gottlieb et al. [5] reported that $81 \%$ of surveyed orthodontists use bonded lingual retainers, of which $37 \%$ use them routinely and $44 \%$ on occasion. However, retainer loss or breakage due to occlusion represents a major problem. Several authors have found that thin wires with less strands showed higher failure rates than wires with more strands or greater thickness $[6,7]$. Increasing the adhesion of the retainer to the teeth has presented a major challenge.

Later developments produced wires made of fibre glass, polyethylene or Kevlar whose flexible fibres were fixated with composite. The benefits of this system include improved wearing comfort and esthetics [8,9]. However, a clinical study by Littlewood et al. [10] showed a 3-year dropout rate of $50 \%$ for fibre-reinforced wires, whereas wires made of stainless steel had a failure rate of only $10 \%$. 
The aim of our study was to compare the SBS of two metal retainers and one fibre-reinforced retainer in combination with three different adhesive systems and to test the effect of sandblasting enamel surfaces before bonding.

\section{Materials and methods}

For this study, we used 400 freshly extracted, caries-free, and structurally intact mandibular bovine teeth. All procedures were carried out by the same operator for all test samples. The teeth were cleaned and polished for 10 seconds with pumice, disinfected with a $0.5 \%$ chloramine solution and the remaining chloramine was rinsed off to avoid chemical reactions with the adhesive. Test pairs of anatomically equal mandibular incisors were set up with approximal contact and embedded into a standardised form filled with epoxy casting resin. Surfaces were positioned in such a way that the retainer could be shorn off parallel to the crown (Figures 1 and 2). The specimens were divided into 10 groups of 20 units each. $50 \%$ of the specimens were sandblasted (KaVo PROPHYflex ${ }^{\oplus}, \mathrm{KaVo}$ Dental GmbH, Biberach/Riss, Germany) with a fluoride-free aluminium oxide powder at an angle of $45^{\circ}$ and a distance of $5 \mathrm{~mm}$ comparable to Cal-Neto et al. [11] to examine the influence of surface roughness on SBS.

Before bonding the enamel of all teeth was etched with $37 \%$ phosphoric acid (Total Etch ${ }^{\mathrm{Ts}}$, Ivoclar Vivadent $\mathrm{GmbH}$, Ellwangen, Germany) for 30 seconds. After the standardised appliance of a thin layer of unfilled resin (Transbond $^{\mathrm{TM}}$ XT Primer, $3 \mathrm{M}$ Unitek AG, Monrovia, CA, USA in the Tetric EvoFlow ${ }^{\mathrm{TM}}$ and Transbond ${ }^{\mathrm{TM}}$ LR groups and Stick Resin ${ }^{\mathrm{Tm}}$, Stick Tech Ltd., Turku, Finland, in the Stick $^{\circ}$ FLOW groups) the wire was bonded with composite and light-cured with a standard curing light
(Elipar $^{\mathrm{TM}}$, 3 M Espe AG, Neuss, Germany) for 40 seconds. For bonding, we used three different composites: Transbond $^{\mathrm{TM}}$ LR (3 M Unitek AG, Monrovia, CA, USA), a highly filled light-cured resin; Tetric EvoFlow ${ }^{\mathrm{Tm}}$ (Ivoclar Vivadent $\mathrm{GmbH}$, Ellwangen, Germany), a flowable light-cured nanohybrid composite and Stick ${ }^{\circ}$ FLOW (Stick Tech Ltd., Turku, Finland), a flowable light-cured microhybrid composite. The latter was used exclusively in conjunction with the everStick ${ }^{\oplus}$ ORTHO fibre-reinforced retainer and vice versa, since they were specifically developed for combined use and Stick ${ }^{\circ}$ FLOW is not recommended for usage in conjunction with steel retainers.

Three different retainers were used in this study: BondA-Braid $^{\text {TM }}$ (Reliance Orthodontic Products Inc., Itasca, USA), an 8-times braided stainless steel retainer mea-

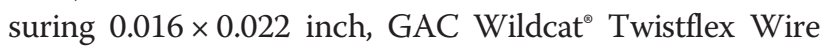
(Ortho-Care Ltd., Bradford, UK), a 3-strand twisted stainless steel retainer measuring 0.0195 inch and everStick ${ }^{\oplus}$ ORTHO (Stick Tech Ltd., Turku Finland), a fibrereinforced retainer consisting of 1000 single unidirectional fibre-reinforced strings coated with PMMA and bis-GMA with a diameter of $0.75 \mathrm{~mm}$. To standardise the bonding procedure, all retainers had a length of $15 \mathrm{~mm}$. To achieve a standardised experimental procedure, we used a custom-made flexible form to position the retainer guaranteeing bonding surfaces of $4 \mathrm{~mm}$ in diameter located $4 \mathrm{~mm}$ apart from each other (Figure 1).

Each specimen was stored in distilled water for 7 days before testing. For debonding we used a universal testing machine (Instron 5965, Instron, Pfungstadt, Germany) at a crosshead speed of $1 \mathrm{~mm} / \mathrm{min}$ (Figure 1). The applied force was directed along the occluso-apical axis of the incisors to simulate the initial bite force. The edge of the shearing rod was positioned in the middle of the interdental

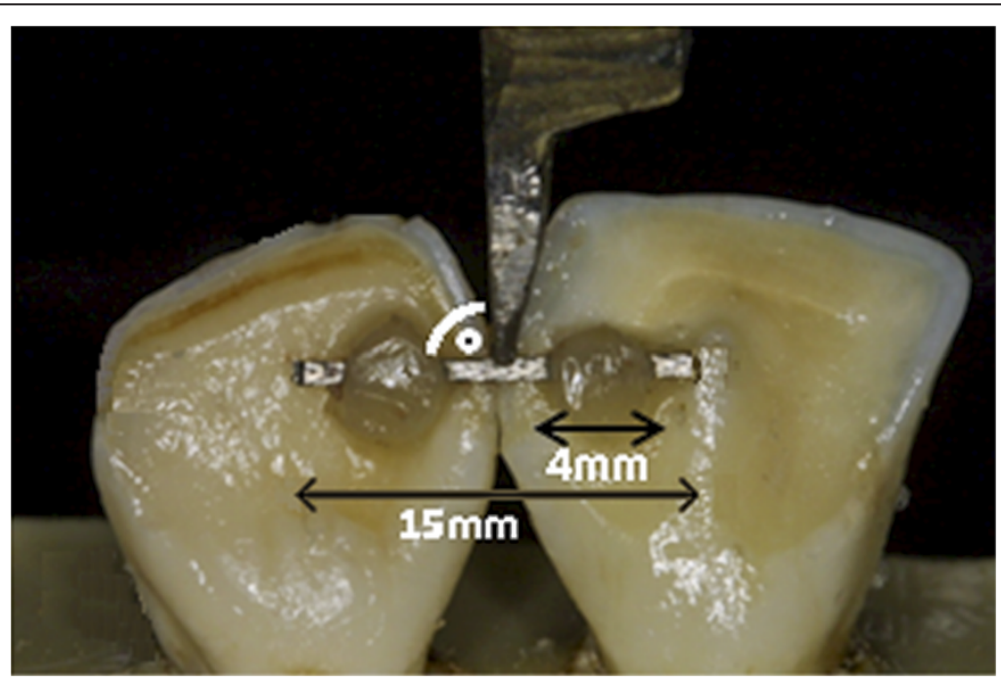

Figure 1 Experimental design for testing of shear bond strength (SBS). The force applied by the universal testing machine Instron 5965 (Instron, Pfungstadt, Germany) was directed along the occluso-apical axis of the incisors to simulate the initial bite force. 


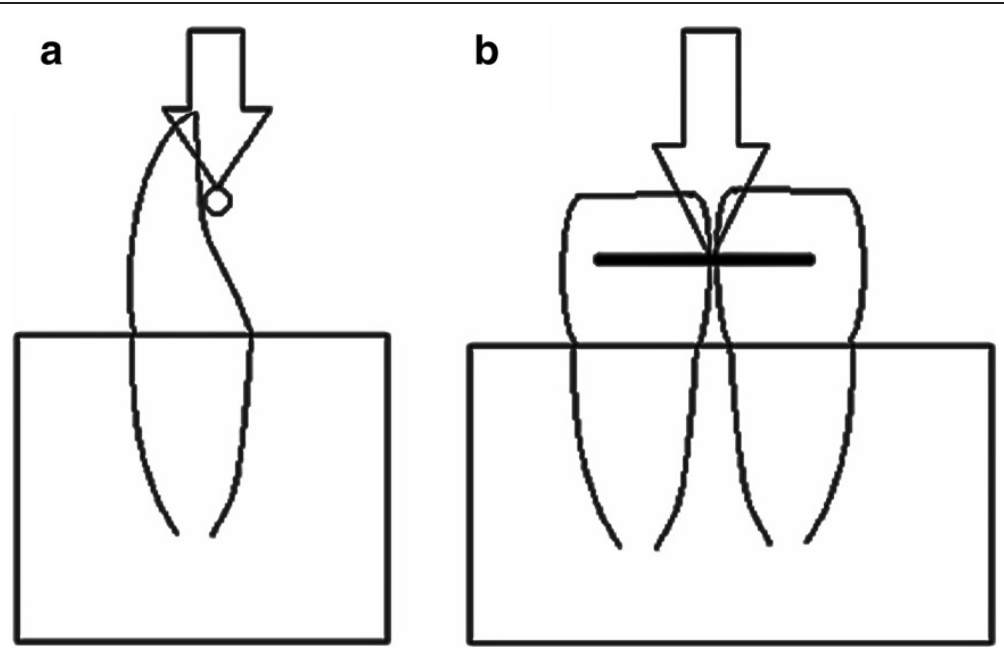

Figure 2 Schematic drawing of experimental design. a view from the side; $\mathbf{b}$ view from oral.

segment. The load on the wire was raised until debonding occurred, and SBS was recorded in Newton (N).

The statistical analysis was performed with the software IBM SPSS Statistics $21^{\circ}$ (IBM, Ehningen, Germany). A two-way independent factorial ANOVA was applied to compare the effect of the different retainer-adhesivecombinations and sandblasting on shear bond strength (SBS), followed-up by bootstrapped Bonferroni post hoc tests (1000 samples) for pairwise comparisons. Since assumptions for parametric tests were partly violated, the post-hoc tests were bootstrapped and nonparametric tests (Mann-Whitney U test and Kruskal-Wallis $\mathrm{H}$ test) used to confirm the ANOVA main effects. A p-value $<0.05$ was deemed significant in all cases. To assess clinical importance, effect sizes were calculated as Pearson's correlation coefficient $r$ with $r>0.5$ constituting a large, $r>0.3$ a medium and $r>0.1$ a small effect/mean difference.

\section{Results}

The highest value for the mean shear bond strength $(\mathrm{M}=156.33 \mathrm{~N} ; \mathrm{SD}=36.40 \mathrm{~N})$ was found for the adhesive Transbond ${ }^{\mathrm{TM}} \mathrm{LR}$ at pretreated surfaces in combination with the retainer wire Bond-A-Braid ${ }^{\text {ms }}$ (group 10) and the second highest value in combination with the retainer GAC Wildcat $^{\oplus}$ Twistflex Wire $(\mathrm{M}=146.11 \mathrm{~N} ; \mathrm{SD}=55.03 \mathrm{~N})$ (group 8) with sandblasting (Table 1, Figure 3). Tetric EvoFlow $^{\mathrm{Tm}}$ showed third best results at pretreated surfaces in combination with the retainer wire Bond-A-Braid ${ }^{\text {TM }}$ $(\mathrm{M}=106.55 \mathrm{~N} ; \mathrm{SD}=21.53 \mathrm{~N})$ (group 6) and the fourth best results in combination with GAC Wildcat ${ }^{\oplus}$ Twistflex Wire $(\mathrm{M}=73.26 \mathrm{~N} ; \mathrm{SD}=27.40 \mathrm{~N})$ (group 4). The combination Bond-A-Braid ${ }^{\mathrm{Tm}} /$ Tetric EvoFlow ${ }^{\mathrm{TM}}$ without sandblasted surfaces (group 5) showed the lowest SBS $(\mathrm{M}=33.55 \mathrm{~N} ; \mathrm{SD}=13.85 \mathrm{~N})$ and the combination Stick $^{\circ}$ FLOW and everStick ${ }^{\ominus}$ ORTHO $(\mathrm{M}=37.02 \mathrm{~N}$;

Table 1 Shear bond strengths (SBS) of the retainer-adhesive-combinations tested with and without sandblasting

\begin{tabular}{|c|c|c|c|c|c|c|c|c|}
\hline Grp & Retainer wire & Bonding & $\mathbf{n}$ & pre & SBS M [N] & SBS min. [N] & SBS max. [N] & SBS SD N] \\
\hline 1 & everStick ${ }^{\circledast}$ ORTHO & Stick FLOW & 20 & no & 37.02 & 11.22 & 81.67 & 14.79 \\
\hline 2 & everStick ${ }^{\circledast}$ ORTHO & Stick ${ }^{\oplus} \mathrm{FLW}$ & 20 & yes & 65.62 & 26.13 & 101.70 & 22.13 \\
\hline 3 & Wildcat ${ }^{\oplus}$ Twistflex Wire & Tetric EvoFlow ${ }^{T M}$ & 20 & no & 44.79 & 15.63 & 114.85 & 22.07 \\
\hline 4 & Wildcat ${ }^{\oplus}$ Twistflex Wire & Tetric EvoFlow ${ }^{\mathrm{TM}}$ & 20 & yes & 73.26 & 24.96 & 122.99 & 27.40 \\
\hline 5 & Bond-A-Braid ${ }^{\mathrm{TM}}$ & Tetric EvoFlow ${ }^{T M}$ & 20 & no & 33.55 & 12.26 & 59.94 & 13.85 \\
\hline 6 & Bond-A-Braid ${ }^{\top \mathrm{M}}$ & Tetric EvoFlow ${ }^{T M}$ & 20 & yes & 106.55 & 63.60 & 143.52 & 21.53 \\
\hline 7 & Wildcat ${ }^{\oplus}$ Twistflex Wire & Transbond $^{\mathrm{TM}} \mathrm{LR}$ & 20 & no & 63.84 & 31.78 & 128.82 & 29.10 \\
\hline 8 & Wildcat ${ }^{\oplus}$ Twistflex Wire & Transbond ${ }^{T M} L R$ & 20 & yes & 146.11 & 25.49 & 258.10 & 55.03 \\
\hline 9 & Bond-A-Braid ${ }^{\top M}$ & Transbond ${ }^{T M} L R$ & 20 & no & 73.22 & 16.94 & 185.11 & 33.06 \\
\hline 10 & Bond-A-Braid $^{\mathrm{TM}}$ & Transbond ${ }^{T M} L R$ & 20 & yes & 156.33 & 72.58 & 209.77 & 36.40 \\
\hline
\end{tabular}

$\mathrm{M}=$ mean; $\mathrm{SD}=$ standard deviation; $\max .=$ maximum; $\min .=$ minimum; Grp = test group; pre = pretreatment (enamel sandblasting). 


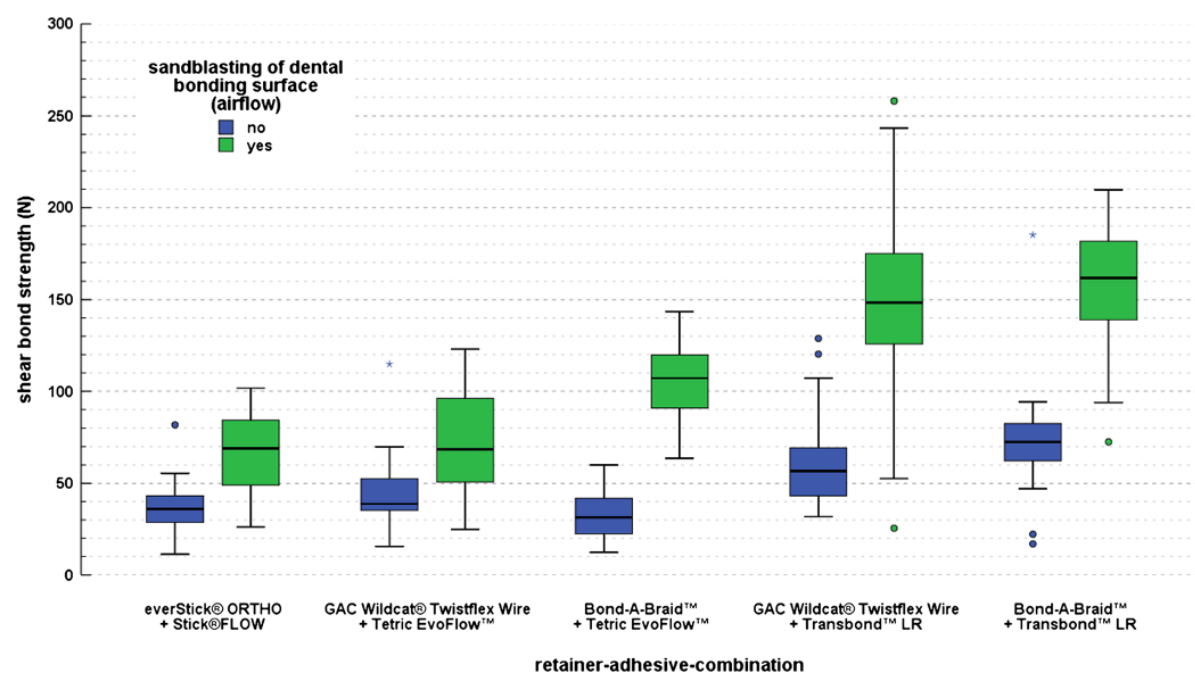

Figure 3 Shear bond strengths (SBS) of the retainer-adhesive-combinations tested with and without prior sandblasting. Boxplots show median and interquartile range while whiskers denote the data range. ${ }^{\circ}$ outliers ( $>1.5 \times$ IQR beyond upper/lower quartile); ${ }^{*}$ extreme values (>3 $\times$ IQR beyond upper/lower quartile).

$\mathrm{SD}=14.79 \mathrm{~N})$ without sandblasted surfaces (group 1) showed the second lowest SBS.

Maximum shear bond strength values were found for the combinations Bond-A-Braid ${ }^{\mathrm{m}} /$ Transbond $^{\mathrm{m}}$ LR $(209.77 \mathrm{~N})$ and GAC Wildcat ${ }^{\circ}$ Twistflex Wire/Transbond ${ }^{\mathrm{mw}}$ LR (258.10 $\mathrm{N}$ ), both at pretreated surfaces (Table 1).

The two-way independent factorial ANOVA showed a significant and large main effect of sandblasting on the measured shear bond strength, $\mathrm{p}<0.001, \mathrm{r}=0.62$, i.e. mean shear bond strength was significantly higher by $217 \%$, when the dental surfaces were sandblasted prior to bonding. This was confirmed by a Mann-Whitney-U test (Table 2).

There was a significant and large ANOVA main effect of the retainer-adhesive-combination used on the measured SBS, $\mathrm{p}<0.001, \mathrm{r}=0.73$, i.e. the tested combinations differed significantly in SBS. This was confirmed by a nonparametric Kruskal-Wallis-H test $(n=200, \mathrm{p}<0.001)$. The bootstrapped Bonferroni post-hoc tests showed that after surface preconditioning the combinations GAC Wildcat ${ }^{\circ}$ Twistflex Wire/Tetric EvoFlow $^{\text {mam }}$ and everStick ${ }^{\circ}$ ORTHO/ Stick ${ }^{\circ}$ FLOW did not perform significantly different when measuring SBS $(\mathrm{p}=0.337)$, as was the case with the combinations GAC Wildcat ${ }^{\circ}$ Twistflex Wire /Transbond ${ }^{\mathrm{Tm}} \mathrm{LR}$

Table 2 Sandblasting significantly increased mean shear bond strength (Mann-Whitney-U-test)

\begin{tabular}{llllllll}
\hline Sandblasting & $\mathbf{n}$ & $\mathbf{M}$ & $\mathbf{S D}$ & $\mathbf{U}$ & $\mathbf{z}$ & $\mathbf{p}$ & $\mathbf{r}$ \\
\hline Yes & 100 & 109.57 & 28.04 & 8581 & 8.75 & $<0.001$ & 0.62 \\
No & 100 & 50.49 & 50.32 & & & & \\
\hline
\end{tabular}

$\mathrm{n}=$ number of tested specimens; $\mathrm{M}=$ mean; $\mathrm{SD}=$ standard deviation; $\mathrm{U}=$ test statistic Mann-Whitney-U-test; $\mathrm{z}=$ standardized test statistic (z-score); $p=$ significance level; $r=$ Pearson's correlation coefficient (effect size). and Bond-A-Braid ${ }^{\mathrm{Tw}} /$ Transbond $^{\mathrm{Tw}}$ LR ( $\left.\mathrm{p}=0.488\right)$. Without surface preconditioning similar results were obtained, except for the combination Bond-A-Braid ${ }^{\mathrm{mw}} /$ Tetric EvoFlow ${ }^{\mathrm{mex}}$ that did not yield significantly higher SBS values than the GAC Wildcat ${ }^{\circ}$ Twistflex Wire/Tetric EvoFlow ${ }^{\mathrm{Tm}}$ combina- $^{-}$ tion (Figure 3, Table 1). Sandblasting significantly affected the performance of the retainer-wire-combinations in relation to each other (ANOVA $\mathrm{p}<0.001, \mathrm{r}=0.29$ ).

The metal wire combinations bonded with Transbond $\mathrm{LR}^{\mathrm{Tw}}$ showed significantly higher SBS than the wire combinations bonded with Tetric EvoFlow ${ }^{\mathrm{ma}}$, independent of the pretreatment process and the retainer wire used. However, when Tetric EvoFlow ${ }^{\text {ma }}$ was used, Bond-A-Braid ${ }^{\mathrm{m}}$ showed significantly higher SBS than GAC Wildcat ${ }^{\circ}$ Twistflex Wire at pretreated surfaces $(\mathrm{p}=0.001)$.

\section{Discussion}

In the present study we simulated the clinical bite situation by putting a vertical thrust on the retainer. Reynolds et al. [12] and Reicheneder et al. [13] found that a vertical thrust yields the highest values of SBS compared to a tensile force in horizontal or vertical orientation. However, SBS not only depends on the direction, but also on the location of the applied force. Several authors have demonstrated that the lowest values of SBS occur when the force is applied to the interdental segment $[8,14]$. Therefore we chose this most fragile segment to determine the lowest strength required for debonding.

In this study bovine teeth were used. Hobson et al. [15] recommended using incisors instead of human molars and premolars, because they found a significant difference of SBS between different tooth types. However, Nakamichi 
et al. [16] compared the SBS of human teeth versus bovine teeth and figured out that there was no significant difference in the results. Therefore, the use of bovine teeth seemed most suitable for our experimental purposes.

Faltermeier et al. [17] found that the SBS of two- and three-component adhesives significantly exceed that of one bottle systems. Thus in our study we only used three-component systems. However, those systems showed significant differences in bonding strength when tested with different retainer wires independent of surface pretreatment.

Zachrisson [18] and Oesterle et al. [19] enlarged the surfaces of both ends of two wires measuring 0.030 inch and 0.032 inch by sandblasting to increase the adhesion between metal and composite. We investigated the influence of sandblasting the dental bonding surface on the SBS of the retainer. The results showed that the SBS increased for all retainer-wire-combinations tested with a grand total of $217 \%$. This indicates that sandblasting has a profound effect on clinical stability of the bonded retainer because of increased micro-retention after etching. This was confirmed by Reisner et al. [20], who examined the influence of enamel preparation on the SBS of orthodontic brackets by differentiating four groups: only sandblasted, sandblasted before etching, only acid etched and buffed with a fluted bur. They also concluded that sandblasting was no substitute for acid etching. Cal-Neto et al. [11] tested the effect of intraoral sandblasting prior to enamel etching. The bond strength increased, but the log-rank test did not show any significantly different clinical performance. In general, sandblasting not only increases the roughness of teeth, but also guarantees for a clean surface free from plaque and debris requiring only little efforts of time and material.

In our study, the Bond-A-Braid ${ }^{\mathrm{TM}} /$ Transbond $^{\mathrm{TM}}$ LR combination showed the highest SBS value of all combinations, followed by GAC Wildcat ${ }^{\oplus}$ Twistflex Wire/Transbond ${ }^{\mathrm{TM}}$ LR. These results indicate that the bonding strength and clinical stability of Transbond ${ }^{\mathrm{TM}}$ LR are higher than that of all other adhesive systems tested regardless of the retainer wire used.

Furthermore, the results demonstrate that the 8-times braided steel wire (Bond-A-Braid ${ }^{\mathrm{TM}}$ ) tolerated significantly higher SBS compared to the three-stranded wrapped steel wire (GAC Wildcat ${ }^{\oplus}$ Twistflex Wire) when used in combination with Tetric EvoFlow ${ }^{\mathrm{TM}}$, but not with Transbond $^{\mathrm{TM}}$ LR. This indicates that an increased strand count of the retainer wire has generally positive effects on its clinical stability. However, since this was only true for the bonding Tetric EvoFlow ${ }^{\mathrm{Tm}}$ and not for Transbond ${ }^{\mathrm{Tm}} \mathrm{LR}$, we suppose that the influence of the bonding system on SBS is far greater than that of the retainer wire used. The SBS observed by Aldrees et al. [8] in a similar experimental set-up without prior sandblasting and using a 0.0215 inch five-stranded wrapped wire $(\mathrm{M}=70.0 \mathrm{~N}, \mathrm{SD}=$ $14.1 \mathrm{~N})$ were similar to our values obtained from the woven $0.016 \times 0.022$ inch Bond-A-Braid ${ }^{\mathrm{TM}}$ wire without prior sandblasting $(\mathrm{M}=73.2 \mathrm{~N}, \mathrm{SD}=33.1 \mathrm{~N})$. This shows that other factors, such as the diameter of the wire or the amount of its windings, may be decisive for SBS.

Despite yielding excellent aesthetics, the fibre-reinforced retainers showed the lowest SBS of all tested combinations. This was also found by Foek et al. [21], who investigated the shear bond strength of steel wires compared to fibre glass wires. However, everStick ${ }^{\bullet}$ ORTHO fibre glass wire proved to be easy to handle and the observed SBS were not significantly different from those of GAC Wildcat $^{\odot}$ Twistflex Wire in combination with Tetric EvoFlow $^{\mathrm{Tm}}$. Furthermore, fibre glass retainers do not have to be adjusted to the dental arch prior to bonding in contrast to steel retainers. This eliminates the risk of inadvertent orthodontic force application on the retainer teeth.

Reynolds [12] determined that materials for acceptable clinical use in orthodontic treatment should be able to resist forces of 6-8 N. Waters [22] noticed that the normal range of oral forces is 3-18 N. In our study the SBS of all tested retainer systems including the fibrereinforced systems exceeded these values and should therefore show clinically sufficient shear bond strengths.

\section{Conclusions}

- A significant increase of the SBS could be achieved by enamel sandblasting.

- Transbond $^{\mathrm{Tx}}$ LR showed the best results of all the tested adhesives.

- Bond-A-Braid ${ }^{\mathrm{TM}}$ showed higher SBS and thus a lower failure rate in comparison to all other retainers tested.

- All wire/composite combinations tested provided sufficient bonding strengths for clinical use.

\section{Competing interests}

The authors declare that they have no competing interests.

\section{Authors' contributions}

$\mathrm{CR}, \mathrm{BH}, \mathrm{AF}, \mathrm{PP}, \mathrm{CL}$ and $\mathrm{CK}$ contributed to the conception, design and coordination of the study as well as the acquisition of data. CK performed the statistical calculations. CR and CK drafted and wrote the manuscript. CR, $\mathrm{BH}, \mathrm{AF}, \mathrm{PP}, \mathrm{CL}$ and $\mathrm{CK}$ revised the manuscript. All authors read and approved the final manuscript.

\section{Sources of support}

The study was funded by the Department of Orthodontics, University Medical Centre of Regensburg, which - as an public institution - was not involved in study design, data collection, analysis and interpretation or the writing of the article.

\section{Author details}

'Department of Orthodontics, University Medical Centre of Regensburg, Franz-Josef-Strauß-Allee 11, 93053 Regensburg, Germany. ${ }^{2}$ Department of Orthodontics, University of Muenster, Waldeyerstraße 30, 48149 Muenster, Germany. 


\section{References}

1. Little RM, Wallen TR, Riedel RA: Stability and relapse of mandibular anterior alignment-first premolar extraction cases treated by traditional edgewise orthodontics. Am J Orthod Dentofacial Orthop 1981, 80:349-365.

2. Little RM: Stability and relapse of dental arch alignment. Br J Orthod 1990, 7:235-241.

3. Cobourne MT, DiBiase AT: Handbook of Orthodontics. Edinburgh, New York: Mosby; 2009.

4. Zachrisson BU: Clinical experience with direct-bonded orthodontic retainers. Am J Orthod Dentofacial Orthop 1977, 71:440-448.

5. Gottlieb EL, Nelson AH, Vogels DS: JCO study of orthodontic diagnosis and treatment procedures. Part 1. Results and trends. J Clin Orthod 1996, 30:615-629.

6. Artun J, Spadafora AT, Shapiro PA: A 3-year follow-up study of various types of orthodontic canine-to-canine retainers. Eur J Orthod 1997, 19:501-509.

7. Dahl EH, Zachrisson BU: Long-term experience with direct-bonded lingual retainers. J Clin Orthod 1991, 25:619-630.

8. Aldrees AM, Al-Mutairi TK, Hakami ZW, Al-Malki MM: Bonded orthodontic retainers: a comparison of initial bond strength of different wire-and-composite combinations. J Orofac Orthop 2010, 71:290-299.

9. Diamond M: Resin fiberglass bonded retainer. J Clin Orthod 1987, 21:182-183.

10. Littlewood SJ, Millett DT, Doubleday B, Bearn DR, Worthington HV: Orthodontic retention: a systematic review. J Orthod 2006, 33:205-212

11. Cal-Neto JP, Castro S, Moura PM, Ribeiro D, Miguel JAM: Influence of enamel sandblasting prior to etching on shear bond strength of indirectly bonded lingual appliances. Angle Orthod 2011, 81:149-152.

12. Reynolds IR: A review of direct orthodontic bonding. Br J Orthod 1975, 2:171-178.

13. Reicheneder CA, Gedrange T, Lange A, Baumert U, Proff P: Shear and tensile bond strength comparison of various contemporary orthodontic adhesive systems: an in-vitro study. Am J Orthod Dentofacial Orthop 2009 135:422. e1-6; discussion 422-3.

14. Radlanski RJ, Zain ND: Stability of the bonded lingual wire retainer - a study of the initial bond strength. J Orofac Orthoped 2004, 65:321-335.

15. Hobson RS, McCabe JF, Hogg SD: Bond strength to surface enamel for different tooth types. Dent Mat 2001, 17:184-189.

16. Nakamichi I, Iwaku M, Fusayama T: Bovine teeth as possible substitutes in the adhesion test. J Dent Res 1983, 62:1076-1081.

17. Faltermeier A, Behr M, Müssig D: A comparative evaluation of bracket bonding with 1-, 2-, and 3-component adhesive systems. Am J Orthod Dentofacial Orthop 2007, 132:144. e1-5.

18. Zachrisson BJ: Third-generation mandibular bonded lingual 3-3 retainer. J Clin Orthod 1995, 29:39-48.

19. Oesterle LJ, Shellhart WC, Henderson S: Enhancing wire-composite bond strength of bonded retainers with wire surface treatment. Am J Orthod Dentofacial Orthop 2001, 119:625-631.

20. Reisner KR, Levitt HL, Mante F: Enamel preparation for orthodontic bonding: a comparison between the use of a sandblaster and current techniques. Am J Orthod Dentofacial Orthop 1997, 111:366-373.

21. Foek DLS, Ozcan M, Krebs E, Sandham A: Adhesive properties of bonded orthodontic retainers to enamel: stainless steel wire vs fiber-reinforced composites. J Adhes Dent 2009, 11:381-390

22. Waters NE: Some mechanical and physical properties of teeth. Sym Soc Exp Biol 1980, 34:99-135.

doi:10.1186/1746-160X-10-51

Cite this article as: Reicheneder et al:: Shear bond strength of different retainer wires and bonding adhesives in consideration of the pretreatment process. Head \& Face Medicine 2014 10:51.

\section{Submit your next manuscript to BioMed Central and take full advantage of:}

- Convenient online submission

- Thorough peer review

- No space constraints or color figure charges

- Immediate publication on acceptance

- Inclusion in PubMed, CAS, Scopus and Google Scholar

- Research which is freely available for redistribution 